\title{
Direct Medical Expenditures and Healthcare Utilizations in US Hispanic vs. Non-Hispanic Patients with Chronic Obstructive Pulmonary Disease (COPD): An Analysis of the Medical Expenditure Panel Survey 2012-2016
}

\author{
Christian Monzon ${ }^{1 \#}$, Mrudula Raparla ${ }^{1 \#}$, Jihaeng $\mathrm{Heo}^{2}$, Dong Yeong Shin ${ }^{3}$ and Jongwha Chang ${ }^{1 *}$ \\ ${ }^{1}$ Department of Pharmacy Practice, University of Texas, School of Pharmacy, EI paso, TX, USA \\ ${ }^{2}$ Genesis Research, Hoboken, NJ, USA
}

${ }^{3}$ Department of Public Health Sciences, New Mexico State University, Las Cruces, NM, USA

${ }^{\#}$ Both authors contributed equally to this work.

*Corresponding author: Jongwha Chang, Department of Pharmacy Practice, University of Texas, School of Pharmacy, EI Paso, TX, USA

\section{ARTICLE INFO}

Received: 幽 July 18, 2020

Published: 絈 July 31, 2020

Citation: Christian M, Mrudula R, Jihaeng H, Dong Yeong S, Jongwha C. Direct Medical Expenditures and Healthcare Utilizations in US Hispanic vs. Non-Hispanic Patients with Chronic Obstructive Pulmonary Disease (COPD): An Analysis of the Medical Expenditure Panel Survey 2012-2016. Biomed J Sci \& Tech Res 29(2)-2020. BJSTR. MS.ID.004771.

Keywords: Healthcare cost; Healthcare Utilization; COPD; Hispanic Population

Abbreviations: COPD: Chronic Obstructive Pulmonary Disorder; MEPS: Medical Expenditure Panel Survey; AHRQ: Agency for Healthcare Research and Quality; MPC: Medical Provider Component

\section{ABSTRACT}

Introduction: This study aimed to evaluate how chronic obstructive pulmonary disease incidence, healthcare utilization, and cost differ by Hispanic vs. non-Hispanic population category in the United States.

Methods: The retrospective database analysis was employed using 20122016 Medical Expenditure Panel Survey (MEPS) data for patients with COPD. Study outcomes were COPD incidence, healthcare utilization, and healthcare expenditure among Hispanic vs. non-Hispanic population. All-cause direct medical utilization and expenditures were calculated for the following categories of health care services: inpatient hospitalizations, hospital outpatient visits, emergency room visits, officebased physician visits, home health care visits, and prescription medications.

Results: Based on the MEPS data, the total of 11,235 (weighted sample size: $4,869,868)$ patients were included in the analysis after applying inclusion criteria (Hispanic: 2,218 (weighted sample size: 2,363,375) and Non-Hispanic: 9,017 (weighted sample size: 22,506,493)). Hispanic patients had significantly lower expenditures on inpatients visits, hospital outpatients, medications prescriptions compared to NonHispanic patients $(\mathrm{p}<0.001)$. The incremental cost (Non-Hispanic minus Hispanic) was \$ 1,742.83 (95\% CI: 404.35-3,081.32, p=0.011), indicating that Non-Hispanic group had significantly higher cost on overall health care services compared to Hispanic group. Similarly, Non- Hispanic patients showed \$ 764.14 (95\% CI: 453.401,074.89) higher expenditures related COPD than Hispanic patients $(\$ 1,945.61$ (95\% CI: $1,674.63-2,216.58$ ) vs. \$1,181.47 (95\% CI: 950.44-1,412.49). Both inpatients visits and medications prescriptions linked to COPD diagnosis events were significantly higher for Non-Hispanic patients than Hispanic patients.

Conclusion: The results of our study demonstrate that non-Hispanics with COPD have higher healthcare utilization costs compared to Hispanics with COPD. While the overall amount spent on healthcare by Hispanics is less, it emphasizes the need for better management in this subset of the population to minimize the future harm and to establish a fair healthcare system for all Americans. 


\section{Introduction}

Chronic Obstructive Pulmonary Disorder (COPD) has been the fourth leading cause of mortality in the United States since 2014 [1]. Approximately 16 million people in the United States are reported to be experiencing symptoms of COPD [1]. This disease is characterized by two major distinguishing factors: emphysema and chronic bronchitis. Emphysema results from damage to the alveoli in the lungs, and chronic bronchitis refers to long-term inflammation of the bronchial tubes. Patients with COPD have trouble eliminating carbon dioxide from their lungs. The majority of patients experiencing symptoms are on appropriate inhaled medications in order to maintain normal breathing, and proper lung function [2]. According to National Vital Statistics, COPD is projected to move up the list of leading causes of death by 2020 [3]. Tobacco smoking is a prominent precursor of Chronic Obstructive Pulmonary Disorder (COPD) [1,4].

In the United States, the Hispanic population is among the fastest-growing in the nation. Such people have migrated from different countries with various ethnic backgrounds, but regardless, Hispanics are still among the populations most at risk of developing Chronic Obstructive Pulmonary Disorder [1,5]. According to 2015 statistics, $3 \%$ of the US Hispanic population has been diagnosed with COPD [3]. A 9-year update conducted by Diaz concluded that Hispanics have a disproportionate possibility of encountering risk factors, including geographical location, genetic susceptibility and social disparities [4]. Quite often, the minority group discussed reside in rural communities in the United States, possibly exposing them to various environmental chemicals, such as biomass smoke $[3,4]$. The disproportionate prevalence of the disease can be in part attributed to racial ancestry, access to healthcare, and the patient's own disease management perceptions $[2,6,7]$.

In this subset of the population, socio-economic factors could impact the diagnosis and management of COPD [8]. Poor management or delayed diagnosis may significantly increase health care utilization costs. Patients with poorly managed COPD have high all-cause healthcare utilization costs when compared to patients with well-managed COPD [9]. The costs have been increased from $\$ 21,771$ in patients with zero exacerbations to $\$ 47,061$ in patients with three or more exacerbations $[10,11]$. COPD related health care utilization costs has increased by 24 -fold when compared to other medical causes [9]. Low socio-economic groups and lack of health insurance can increase out-of-pocket costs and burden the healthcare system [12]. This widespread disease has led to a substantial increase in costs for patient treatment, according to Dalal, ranging from $\$ 2,003$ to around $\$ 43,000$ per in-patient visit in the United States [13]. In addition to an overall increase in prevalence of the disease, according to previous research done by Borrego, Hispanics had statistically significantly longer in-patient hospital stays. This led to substantially higher costs to the patient [14]. The aim of this study is to analyze and compare the direct medical expenditures and health care utilizations in US Hispanic patients versus Non-Hispanic patients with COPD. This study will establish credible results regarding the disparities between the examined populations, which can then be applied or considered in future studies regarding similar ethnic disparities. Significant findings may support the hypothesis of disproportionate treatment of Hispanics with COPD, which needs addressing and change, in order to establish an equitable healthcare system.

\section{Methods \\ Data Source}

The retrospective database analysis was employed using 20122016 Medical Expenditure Panel Survey (MEPS) data. It was waived by the Institutional Review Board of the University of Texas at El Paso because MEPS is publicly available de-identified database. MEPS is a set of large-scale surveys of families, individuals, their medical providers and employers across the U.S by the Agency for Healthcare Research and Quality (AHRQ). The MEPS has nationally representative estimates of health care use, expenditures, source of payments, and health insurance coverage for the U.S. civilian noninstitutionalized population [15]. It also provides respondents' characteristics such as current health status, demographic and socio-economic characteristics, employment, access to care, and satisfaction with health care. The MEPS includes a new panel of individuals each year that are drawn from a subset of respondents from the previous year's National Health Interview Survey. Each survey panel is overlapped and has five rounds of interviews over a two-year follow-up period and collects self- or caregiver-reported information [16]. This design can provide continuous and current estimates of health care expenditures and utilization at both the person and household level for two panels for each calendar year [17].

MEPS has three major components; the Household Component (HC), the Insurance Component (IC), and the Medical Provider Component (MPC) [18]. For the purposes of this study, only the HC information was used. The HC includes the full-year consolidated data file, medical conditions data file, and the event data files for inpatient hospitalizations, outpatient visits, office-based physician visits, emergency room visits, home health care visits, and prescription medications. For this study, data was pooled from the 2012-2016 MEPS public use files to allow for a large enough sample size and then demographic information, health care utilization and expenditures were extracted.

\section{Patient Selection and Outcomes Measures}

Patients with a diagnosis of COPD and other respiratory disease (International Classification of Diseases, Tenth Revision, Clinical Modification [ICD-10-CM] code: J44, J81, J98; or Clinical Classification Code [CCC]: 127, 129-134) were identified. Only patients who had at least one health care events including inpatient, outpatient, and prescriptions, and aged more than 18 years were 
included in the analyses. The Hispanic and Non-Hispanic population was categorized based on the Hispanic ethnicity variables (HISPANX). The Hispanic population included Puerto Rican, Cuban/ Cuban American, Dominican, Mexican/Mexican American, Central or South American, and Other Latin American. All other patients than Hispanic group were considered Non-Hispanic cohort [19].

All-cause direct medical utilization and expenditures were calculated for the following categories of health care services: inpatient hospitalizations, hospital outpatient visits, emergency room visits, office-based physician visits, home health care visits, and prescription medications. The utilization is a count of the number of medical events reported for the category. The expenditures in MEPS are defined as the sum of direct payments for health care services provided during the year, including outof-pocket payments and payments by private insurance, Medicaid, Medicare, and other sources. Payments for over-the-counter drugs are not included in MEPS data. Because societal perspective was used for the study, all sources were analyzed, including out of pocket by patient or patient's family, Medicare, Medicaid, private Insurance, Veterans' Administration, TRICARE, other federal sources, other state and local source, worker's Compensation, other unclassified source [14]. COPD and other respiratory diseaserelated direct medical utilization and expenditures were summed based on only health care events linked with an ICD-9-CM code of J44, J81, and J98; or CCC of 127, 129-134. The code list of COPD and other respiratory disease was defined base on the category determined by AHRQ [20]. All expenditures were adjusted to 2016 dollars using the Healthcare Consumer Price Index from the Bureau of Labor Statistics [20].

Covariates were created for each mutually exclusive demographic category: age in years $(<18,18-44,56-64)$; gender; race (White, Black, other/multiple races); educational status (no degree or less than high school, high school, some college); region (Northeast, Midwest, South, West); marital status (married, unmarried (widowed, divorced/separated, never married)); smoking; health insurance status (public, private, uninsured); poverty level (poor/low income (less than 200\%), middle income (200\% to less than $400 \%$ ), high income (greater than or equal to 400\%)); body mass index (BMI, underweight/normal less than 24.9, overweight 25.0-29.9, obese more than 30.0); employment; Charlson Comorbidity Index (CCI, 0, 1-2, more than 3).

\section{Statistical Analyses}

Descriptive statistics (t-tests for continuous variables and Rao-Scott chi-square tests for categorical variables) were conducted to compare the Hispanic and Non-Hispanic groups. For all models, total cause and COPD-related expenditures (as a continuous variable) and utilization (as a count variable) was used as the dependent variable, and all covariates were used as the independent variables. The Generalized Linear Model (GLM) analysis to conduct a regression procedure employing a gamma distribution and a log-link function was used to compare the health care expenditures between Hispanic and Non-Hispanic adjusting covariates. To address a large number of zero-expenditures values on event including inpatients, emergency, and hospital outpatients, and home health care, a two-part model was used to determine the cost of illness for Hispanic individuals compared with Non-Hispanic patients. The first part of the model is a logistic regression model to estimate the probability of having any type of health care expenditure (zero vs. non-zero expenditures), whereas the second part consisted of a generalized linear model with a gamma distribution and a log link to estimate the expenditures on individuals with positive expenditures. For health care utilization, GLM with a Poisson distribution and a log function was applied and zero-inflated Poisson regression was used to address an excess of zero counts on hospital-based events. The incremental cost or counts was calculated by subtracting an expenditure of Hispanic from that of Non-Hispanic group after controlling for covariates. A modified Park test was used to determine the appropriate model for the analysis [21].

Because MEPS employed complex, multistage sampling design, to represent the overall population, responses of the surveyed individuals need to be weighted by the proportion of the population they represent. The person weights provided in the MEPS were used to derive national estimates [22]. An accurate point estimates and its standard errors for the nationally representative population incorporating the MEPS survey weights was calculated using the 'proc survey' procedure of SAS and the 'svy' procedure of Stata [22]. Since this study spans over 5 years, that is, between 2012 and 2016, to calculate the averaged expenditures and utilizations over the number of years of pooled data, the pooled person weights were divided by 5 [23]. All analyses were conducted using SAS version 9.4 (SAS Institute Inc., Cary, NC) and Stata version 15 (Stata Corp. LP, College Station, TX).

\section{Results}

Based on the MEPS data, 172,564 (weighted sample size: $318,443,439$ ) respondents with positive individual weight were identified from 2012 to 2016. The total of 11,235 (weighted sample size: $24,869,868)$ patients were included in the analysis after applying inclusion criteria (Hispanic: 2,218 (weighted sample size: 2,363,375) and Non-Hispanic: 9,017 (weighted sample size: 22,506,493)) (Figure 1). The mean age (standard deviation [SD]) for study patients was 55.4 (0.33) years. Most patients were female (60.8\%), white (84.0\%), and educated higher than college (58.2\%). More than $75 \%$ of patients do not smoke currently and $53 \%$ of patients married in years of interest. Hispanic group included a lower percentage of private insurance $(50.4 \%)$ than $(67.9 \%)$ $(\mathrm{p}<0.001)$. Hispanic group had a higher percentage of poor or low income $(45.6 \%)$ compared to Non-Hispanic group $(30.5 \%)$ $(\mathrm{p}<0.001)$. Hispanics had higher BMI values, but lower CCI value than Non-Hispanic group (Table 1). 


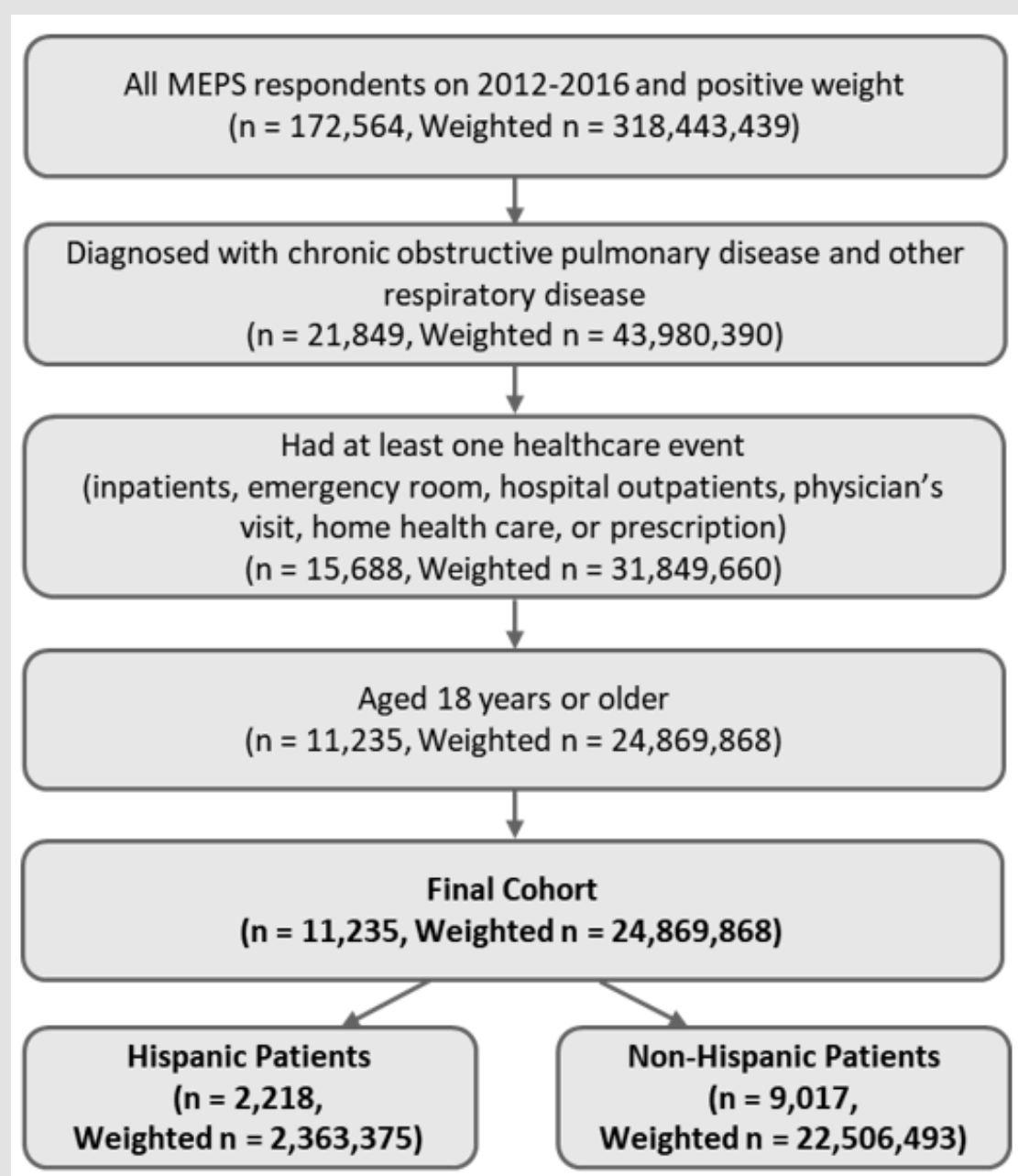

Figure 1: Patient Attrition.

Table 1: Demographics and Clinics Characteristics of COPD and other respiratory disease population.

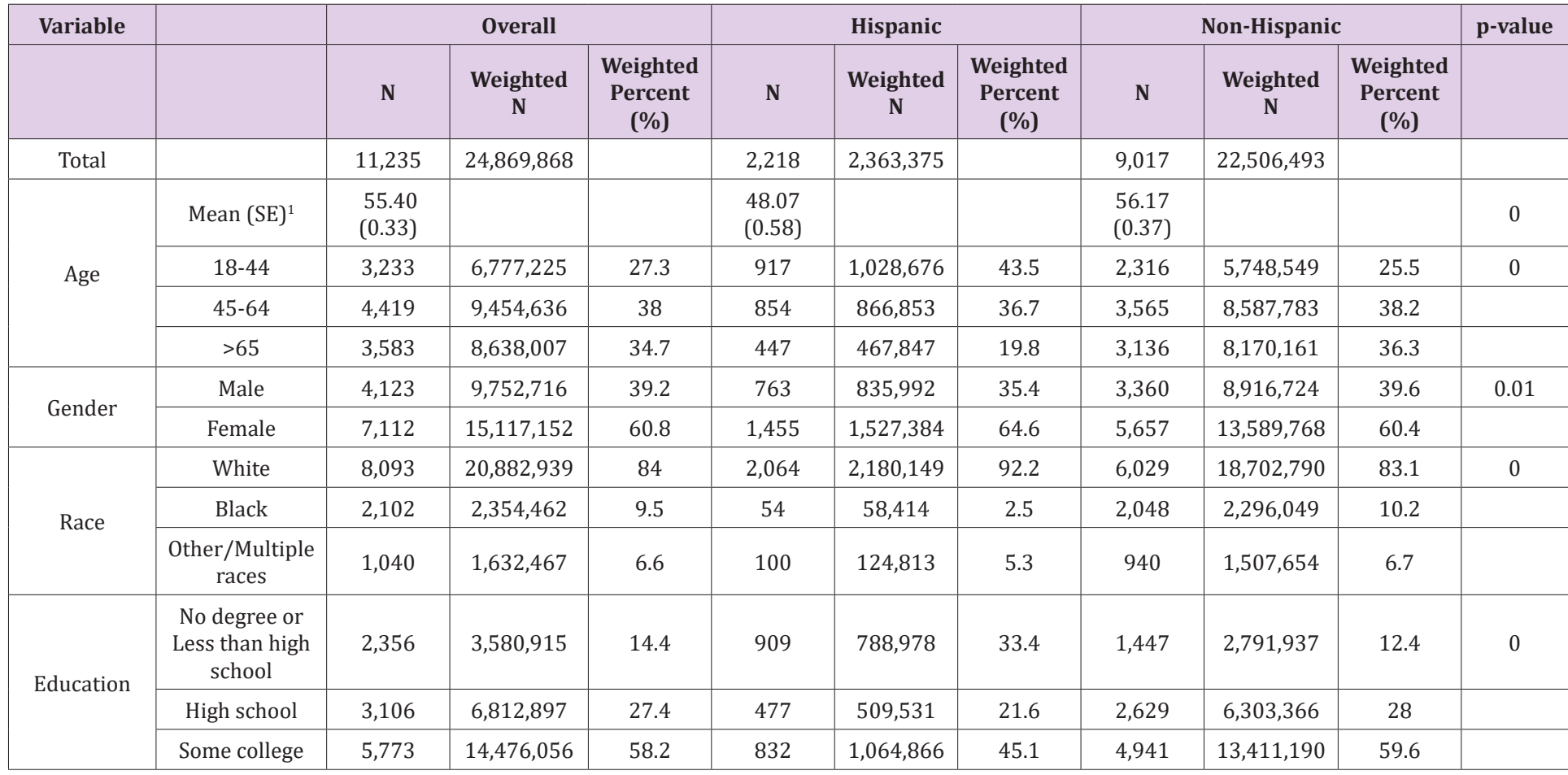




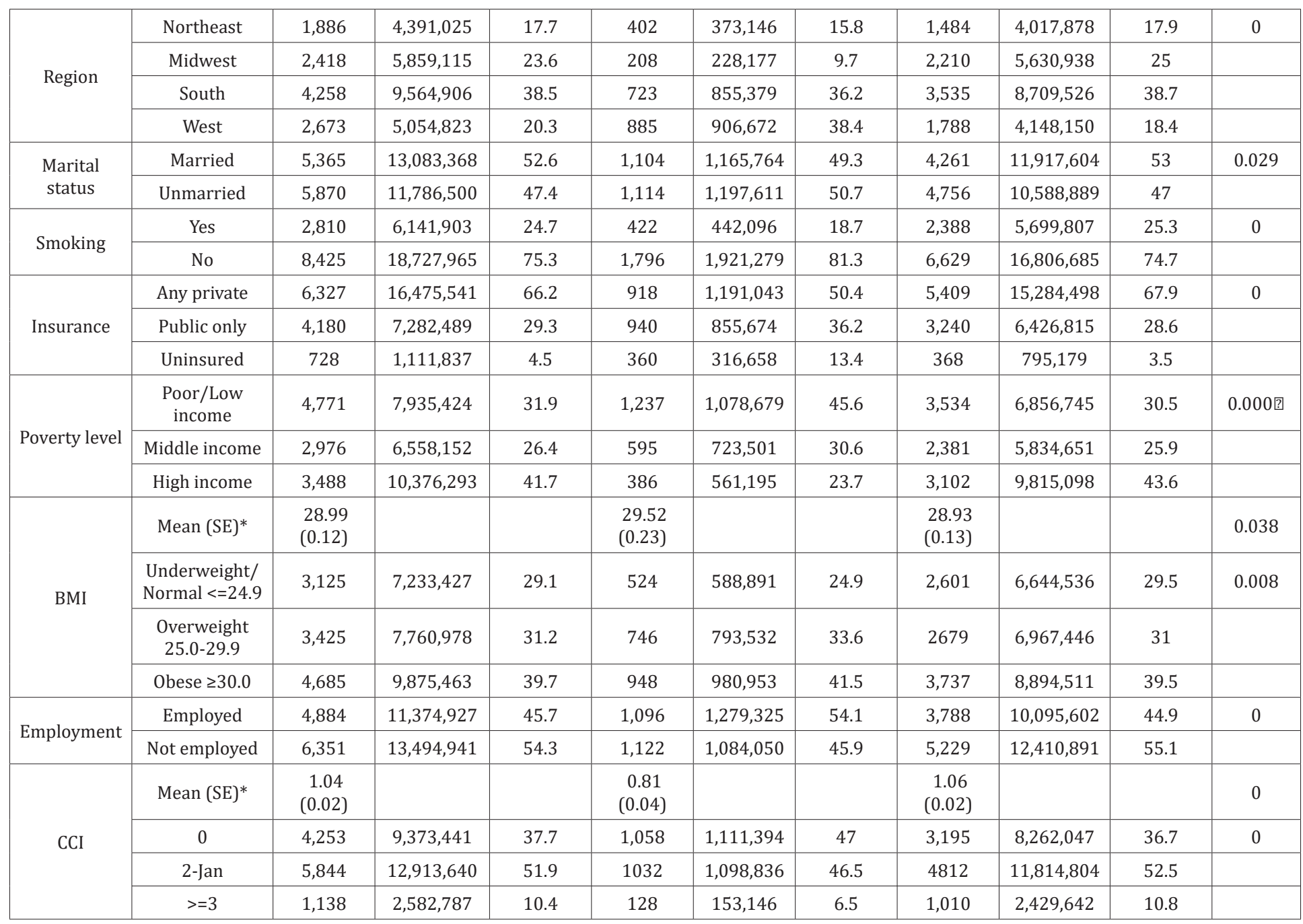

Note: ${ }^{1}$ Continuous mean and SE are for weighted sample.

BMI: Body Mass Index; CCI: Charlson Comorbidity Index; COPD: Chronic Obstructive Pulmonary Disease, SE: Standard Error

Overall all-cause unadjusted medical expenditures in Overall COPD-related expenditures Hispanic patients spent were Hispanic group was \$ 8,986.36 (95\% CI: 7,880.57-10,092.15), significantly lower than those of Non-Hispanic patients (\$1,118.92 while $\$ 12,278.00$ (95\% CI: 11,578.37-12,977.41) was in the Non- $\quad$ (95\% CI: 853.88-1,383.97) vs. \$2,004.46 (95\% CI: 1,789.05Hispanic group $(\mathrm{p}<0.001)$. Hispanic patients had significantly lower $2,219.87), \mathrm{p}<0.001)$. Inpatient visits and prescription cost were expenditures on inpatients visits, hospital outpatients, medications lower in Hispanic compared to the Non-Hispanic group ( $\mathrm{p}<0.001$ ) prescriptions compared to Non-Hispanic patients $(\mathrm{p}<0.001)$. (Table 2). This result was consistent with COPD-related expenditures.

Table 2: Demographics and Clinics Characteristics of COPD and other respiratory disease population.

\begin{tabular}{|c|c|c|c|c|c|c|c|c|c|}
\hline \multirow[b]{2}{*}{ Category $^{1}$} & \multicolumn{4}{|c|}{ Hispanic (\$) } & \multicolumn{5}{|c|}{ Non-Hispanic (\$) } \\
\hline & Mean & SE & $\begin{array}{l}\text { Lower } \\
\mathbf{9 5 \%} \text { CI }\end{array}$ & $\begin{array}{l}\text { Upper } \\
95 \% \text { CI }\end{array}$ & Mean & SE & $\begin{array}{l}\text { Lower } \\
\mathbf{9 5 \%} \text { CI }\end{array}$ & $\begin{array}{l}\text { Upper } \\
95 \% \text { CI }\end{array}$ & p-value ${ }^{2}$ \\
\hline \multicolumn{10}{|c|}{ All-cause expenditures } \\
\hline Overall & $8,986.36$ & 562.69 & $7,880.57$ & $10,092.15$ & $12,278.00$ & 355.96 & $11,578.37$ & $12,977.41$ & $<.0001$ \\
\hline Inpatient & $2,444.75$ & 309.89 & $1,835.75$ & $3,053.75$ & $3,556.77$ & 190.6 & $3,182.20$ & $3,931.33$ & 0.002 \\
\hline Emergency room & 416.45 & 47.12 & 323.84 & 509.06 & 449.43 & 28.46 & 393.5 & 505.36 & 0.542 \\
\hline Hospital outpatient & 524.4 & 81.4 & 364.43 & 684.36 & 948.65 & 63.53 & 823.79 & $1,073.50$ & $<.0001$ \\
\hline $\begin{array}{l}\text { Physician's visits } \\
\text { (Office based visits) }\end{array}$ & $2,323.09$ & 173.93 & $1,981.28$ & $2,664.90$ & $2,683.27$ & 90.63 & $2,505.17$ & $2,861.37$ & 0.069 \\
\hline Home health care & 805.65 & 187.11 & 437.94 & $1,173.35$ & 594.09 & 65.75 & 464.87 & 723.3 & 0.277 \\
\hline
\end{tabular}




\begin{tabular}{|c|c|c|c|c|c|c|c|c|c|}
\hline Prescription & $2,117.27$ & 151.62 & $1,819.31$ & $2,415.23$ & $3,405.84$ & 138.16 & $3,134.34$ & $3,677.34$ & $<.0001$ \\
\hline $\begin{array}{c}\text { COPD-related } \\
\text { expenditures }\end{array}$ & & & & & & & & \\
\hline Overall & $1,118.92$ & 134.43 & 853.88 & $1,383.97$ & $2,004.46$ & 109.26 & $1,789.05$ & $2,219.87$ & $<.0001$ \\
\hline Inpatient & 346.6 & 78.87 & 191.1 & 502.1 & 803.94 & 94.54 & 617.55 & 990.33 & 0 \\
\hline Emergency room & 76.14 & 10.89 & 54.67 & 97.61 & 96.05 & 11.36 & 73.64 & 118.46 & 0.178 \\
\hline Hospital outpatient & 60.91 & 19.41 & 22.64 & 99.17 & 92.57 & 14.98 & 63.04 & 122.1 & 0.21 \\
\hline $\begin{array}{c}\text { Physician's visits } \\
\text { (Office based visits) }\end{array}$ & 312.36 & 59.47 & 195.11 & 429.6 & 299.06 & 13.96 & 271.54 & 326.58 & 0.826 \\
\hline Home health care & 109.14 & 48.26 & 13.99 & 204.28 & 137.87 & 19.17 & 100.08 & 175.65 & 0.571 \\
\hline Prescription & 213.78 & 26.75 & 161.04 & 266.53 & 574.98 & 24.46 & 526.76 & 623.2 & $<.0001$ \\
\hline
\end{tabular}

Note: 1Includes patients with zero-cost for each category.

2Significant at $\mathrm{P}<0.05$ (in bold).

CI: Confidence Interval; COPD: Chronic Obstructive Pulmonary Disease; SE: Standard Error

The GLM with gamma distribution and a log-link function showed that overall all-cause expenditures in Hispanic and NonHispanic was \$10,387.46 (95\% CI: 9,141.80-11,633.12) and $\$ 12,130.29$ (95\% CI: 11,451.86-12,808.72), respectively. The incremental cost (Non-Hispanic minus Hispanic) was $\$ 1,742.83$ (95\% CI: 404.35-3,081.32, p=0.011), indicating that Non-Hispanic group had significantly higher cost on overall health care services compared to Hispanic group. Similarly, Non- Hispanic patients showed \$764.14 (95\% CI: 453.40-1,074.89) higher expenditures related COPD than Hispanic patients (\$1,945.61 (95\% CI: 1,674.63$2,216.58$ ) vs. $\$ 1,181.47$ (95\% CI: 950.44-1,412.49). Both inpatients visits and medications prescriptions linked to COPD diagnosis events were significantly higher for Non-Hispanic patients than Hispanic patients (Table 3). The average of unadjusted number of all-cause inpatients visits were, on average, 0.18 (95\% CI: 0.150.21 ) for Hispanic and 0.26 (95\% CI: 0.24-0.28) for Non-Hispanic groups $(\mathrm{p}<0.001)$, including patients with zero-visits. All-cause hospital outpatients visits, physicians' visits, and medication prescriptions were significantly higher utilization for Non-Hispanic than Hispanic. The COPD-related events in Non-Hispanic group had also a larger number of utilizations on inpatients visits (0.03 vs. $0.06)$, hospital outpatients visits (0.08 vs. 0.15$)$, and medications prescriptions (1.71 vs. 2.15) than Hispanic (Table 4).

Table 3: All-cause and COPD-related adjusted mean and incremental expenditures.

\begin{tabular}{|c|c|c|c|c|c|c|c|c|c|c|c|c|c|}
\hline$\otimes$ & \multicolumn{4}{|c|}{ Hispanic (\$) } & \multicolumn{4}{|c|}{ Non-Hispanic (\$) } & \multicolumn{5}{|c|}{ Incremental cost (Non-Hispanic - Hispanic) (\$) } \\
\hline $\begin{array}{c}\text { Catego- } \\
\text { ry }^{1}\end{array}$ & Mean $^{2}$ & SE & $\begin{array}{l}\text { Lower } \\
\text { 95\% CI }\end{array}$ & $\begin{array}{l}\text { Upper } \\
\text { 95\% CI }\end{array}$ & Mean $^{2}$ & SE & $\begin{array}{l}\text { Lower } \\
\text { 95\% CI }\end{array}$ & $\begin{array}{l}\text { Upper } \\
\text { 95\% CI }\end{array}$ & Mean $^{2}$ & SE & $\begin{array}{l}\text { Lower } \\
\text { 95\% CI }\end{array}$ & $\begin{array}{l}\text { Upper } \\
95 \% \text { CI }\end{array}$ & $\begin{array}{c}\text { p-val- } \\
\text { ue }^{3}\end{array}$ \\
\hline \multicolumn{14}{|c|}{ All-cause expenditures } \\
\hline Overall & $10,387.46$ & 631.84 & $9,141.80$ & $11,633.12$ & $12,130.29$ & 344.12 & $11,451.86$ & $12,808.72$ & $1,742.83$ & 678.92 & 404.35 & $3,081.32$ & 0.011 \\
\hline $\begin{array}{c}\text { Inpa- } \\
\text { tient }\end{array}$ & $2,744.53$ & 358.27 & $2,038.20$ & $3,450.87$ & $3,122.98$ & 170.38 & $2,787.07$ & $3,458.88$ & 378.44 & 383.76 & -378.14 & $1,135.03$ & 0.325 \\
\hline $\begin{array}{l}\text { Emer- } \\
\text { gency } \\
\text { room }\end{array}$ & 449.58 & 56.00 & 339.17 & 559.99 & 438.18 & 27.20 & 384.56 & 491.80 & -11.40 & 56.79 & -123.36 & 100.57 & 0.841 \\
\hline $\begin{array}{c}\text { Hos- } \\
\text { pital } \\
\text { outpa- } \\
\text { tient }\end{array}$ & 616.38 & 82.54 & 453.65 & 779.11 & 931.77 & 57.57 & 818.27 & $1,045.26$ & 315.39 & 102.91 & 112.49 & 518.28 & 0.002 \\
\hline $\begin{array}{l}\text { Physi- } \\
\text { cian's } \\
\text { visits } \\
\text { (Office } \\
\text { based } \\
\text { visits) }\end{array}$ & $2,846.70$ & 187.56 & $2,476.93$ & $3,216.46$ & $2,692.10$ & 87.13 & $2,520.32$ & $2,863.88$ & -154.60 & 187.62 & -524.49 & 215.30 & 0.411 \\
\hline $\begin{array}{l}\text { Home } \\
\text { health } \\
\text { care }\end{array}$ & $1,015.37$ & 242.36 & 537.57 & $1,493.18$ & 547.76 & 58.67 & 432.09 & 663.44 & -467.61 & 240.24 & -941.25 & 6.03 & 0.053 \\
\hline $\begin{array}{l}\text { Pre- } \\
\text { scrip- } \\
\text { tion }\end{array}$ & $2,471.47$ & 171.72 & $2,132.93$ & $2,810.02$ & $3,735.80$ & 161.31 & $3,417.78$ & $4,053.82$ & $1,264.33$ & 195.95 & 878.01 & $1,650.64$ & $<.0001$ \\
\hline
\end{tabular}




\begin{tabular}{|c|c|c|c|c|c|c|c|c|c|c|c|c|c|}
\hline \multicolumn{14}{|c|}{ COPD-related expenditures } \\
\hline Overall & $1,181.47$ & 117.18 & 950.44 & $1,412.49$ & $1,945.61$ & 137.45 & $1,674.63$ & $2,216.58$ & 764.14 & 157.62 & 453.40 & $1,074.89$ & $<.0001$ \\
\hline $\begin{array}{l}\text { Inpa- } \\
\text { tient }\end{array}$ & 381.97 & 102.49 & 179.92 & 584.02 & 634.34 & 87.65 & 461.54 & 807.15 & 252.37 & 124.69 & 6.56 & 498.19 & 0.044 \\
\hline $\begin{array}{l}\text { Emer- } \\
\text { gency } \\
\text { room }\end{array}$ & 81.64 & 14.81 & 52.44 & 110.84 & 90.39 & 10.47 & 69.75 & 111.04 & 8.75 & 12.54 & -15.97 & 33.48 & 0.486 \\
\hline $\begin{array}{c}\text { Hos- } \\
\text { pital } \\
\text { outpa- } \\
\text { tient }\end{array}$ & 92.21 & 29.26 & 34.53 & 149.88 & 91.38 & 14.20 & 63.37 & 119.38 & -0.83 & 30.92 & -61.79 & 60.13 & 0.979 \\
\hline $\begin{array}{l}\text { Physi- } \\
\text { cian's } \\
\text { visits } \\
\text { (Office } \\
\text { based } \\
\text { visits) }\end{array}$ & 334.50 & 40.89 & 253.88 & 415.12 & 300.38 & 14.29 & 272.20 & 328.55 & -34.12 & 39.39 & -111.78 & 43.54 & 0.387 \\
\hline $\begin{array}{c}\text { Home } \\
\text { health } \\
\text { care }\end{array}$ & 107.72 & 49.88 & 9.38 & 206.06 & 130.27 & 21.52 & 87.83 & 172.70 & 22.55 & 53.49 & -82.90 & 128.00 & 0.674 \\
\hline $\begin{array}{l}\text { Pre- } \\
\text { scrip- } \\
\text { tion }\end{array}$ & 323.38 & 38.76 & 246.95 & 399.80 & 609.15 & 28.44 & 553.07 & 665.22 & 285.77 & 44.45 & 198.13 & 373.41 & $<.0001$ \\
\hline
\end{tabular}

Note: ${ }^{1}$ Includes patients with zero-cost for each category.

${ }^{2}$ Adjusted by covariates including age, gender, race, educational status, region, marital status, smoking, health insurance status, poverty level, Body Mass Index, employment, Charlson Comorbidity Index.

${ }^{3}$ Significant at $\mathrm{P}<0.05$ (in bold).

CI: Confidence Interval; COPD: Chronic Obstructive Pulmonary Disease; SE: Standard error

Table 4: All-cause and COPD-related unadjusted healthcare utilization.

\begin{tabular}{|c|c|c|c|c|c|c|c|c|c|}
\hline \multirow[b]{2}{*}{ Category $^{1}$} & \multicolumn{4}{|c|}{ Hispanic } & \multicolumn{4}{|c|}{ Non-Hispanic } & \multirow{2}{*}{ p-value ${ }^{2}$} \\
\hline & Mean & SE & $\begin{array}{l}\text { Lower } \\
\mathbf{9 5 \%} \text { CI }\end{array}$ & $\begin{array}{l}\text { Upper95\% } \\
\text { CI }\end{array}$ & Mean & SE & $\begin{array}{l}\text { Lower } \\
95 \% \text { CI }\end{array}$ & $\begin{array}{l}\text { Upper } \\
95 \% \text { CI }\end{array}$ & \\
\hline \multicolumn{10}{|c|}{ All-cause visits } \\
\hline Inpatient & 0.18 & 0.01 & 0.15 & 0.21 & 0.26 & 0.01 & 0.24 & 0.28 & $<.0001$ \\
\hline Emergency room & 0.41 & 0.03 & 0.36 & 0.46 & 0.46 & 0.02 & 0.43 & 0.49 & 0.116 \\
\hline Hospital outpatient & 0.66 & 0.08 & 0.50 & 0.81 & 1.27 & 0.06 & 1.14 & 1.40 & $<.0001$ \\
\hline $\begin{array}{l}\text { Physician's visits (Office } \\
\text { based visits) }\end{array}$ & 10.77 & 0.75 & 9.29 & 12.25 & 13.03 & 0.27 & 12.49 & 13.56 & 0.005 \\
\hline Home health care & 8.13 & 2.14 & 3.93 & 12.34 & 4.71 & 0.46 & 3.80 & 5.61 & 0.117 \\
\hline Prescription & 25.74 & 2.00 & 21.82 & 29.66 & 32.36 & 0.61 & 31.16 & 33.56 & 0.001 \\
\hline \multicolumn{10}{|c|}{ COPD-related visits } \\
\hline Inpatient & 0.03 & 0.01 & 0.02 & 0.04 & 0.06 & 0.01 & 0.05 & 0.07 & 0.002 \\
\hline Emergency room & 0.11 & 0.01 & 0.09 & 0.13 & 0.10 & 0.01 & 0.09 & 0.12 & 0.810 \\
\hline Hospital outpatient & 0.08 & 0.02 & 0.05 & 0.12 & 0.15 & 0.02 & 0.12 & 0.19 & 0.006 \\
\hline $\begin{array}{l}\text { Physician's visits (Office } \\
\text { based visits) }\end{array}$ & 1.81 & 0.27 & 1.27 & 2.35 & 1.85 & 0.06 & 1.73 & 1.98 & 0.879 \\
\hline Home health care & 0.09 & 0.04 & 0.02 & 0.16 & 0.13 & 0.01 & 0.11 & 0.16 & 0.237 \\
\hline Prescription & 1.71 & 0.07 & 1.57 & 1.85 & 2.15 & 0.04 & 2.07 & 2.22 & $<.0001$ \\
\hline
\end{tabular}

Note: ${ }^{1}$ Includes patients with zero-cost for each category.

2Significant at $\mathrm{P}<0.05$ (in bold).

CI: Confidence Interval, COPD: Chronic Obstructive Pulmonary Disease; SE: Standard error 
Table 5 showed that, after controlling for covariates, all-cause number of visits on home health care were higher in Hispanics than Non-Hispanic group (4.93 vs. 2.57, $\mathrm{p}=0.009$ ). All other averaged number of events did not show the significant different between groups. Regarding the COPD-related events, both inpatients visits and prescriptions showed the significantly higher utilizations for Non-Hispanic than Hispanic groups ( 0.05 vs. $0.03 ; 2.16$ vs. 1.83 , respectively). The incremental visits were 0.02 (95\% CI: 0.00-0.03, $\mathrm{p}=0.017)$ in inpatients visits and 0.32 (95\% CI: 0.14-0.50, p<0.001) in medications prescriptions (Table 5). The GLM of COPD-related expenditures adjusting covariates generated that Hispanic group had significantly lower expenditures than Non-Hispanic group ( $\beta=-$ 0.50 (95\% CI: -0.71- -0.29), p<0.001) (Table 6). A larger CCI was associated with higher health care expenditures $(\beta=0.40(95 \% \mathrm{CI}$ : 0.31-0.49), $\mathrm{p}<0.001$ ) (Table 6).

Table 5: All-cause and COPD-related adjusted mean and incremental healthcare utilization.

\begin{tabular}{|c|c|c|c|c|c|c|c|c|c|c|c|c|c|}
\hline \multirow[b]{2}{*}{ Category $^{1}$} & \multicolumn{4}{|c|}{ Hispanic (\$) } & \multicolumn{4}{|c|}{ Non-Hispanic (\$) } & \multicolumn{5}{|c|}{$\begin{array}{c}\text { Incremental counts (Non-Hispanic - Hispanic) } \\
\text { (\$) }\end{array}$} \\
\hline & Mean $^{2}$ & SE & $\begin{array}{l}\text { Lower } \\
95 \% \mathrm{CI}\end{array}$ & $\begin{array}{c}\text { Upper } \\
95 \% \text { CI }\end{array}$ & Mean $^{2}$ & SE & $\begin{array}{l}\text { Lower } \\
95 \% \text { CI }\end{array}$ & $\begin{array}{l}\text { Upper } \\
95 \% \text { CI }\end{array}$ & Mean $^{2}$ & SE & $\begin{array}{l}\text { Lower } \\
95 \% \text { CI }\end{array}$ & $\begin{array}{l}\text { Upper } \\
95 \% \text { CI }\end{array}$ & $\begin{array}{c}\text { p-val- } \\
\text { ue }^{3}\end{array}$ \\
\hline \multicolumn{14}{|c|}{ All-cause visits } \\
\hline Inpatient & 0.20 & 0.02 & 0.16 & 0.24 & 0.23 & 0.01 & 0.21 & 0.25 & 0.03 & 0.02 & -0.01 & 0.07 & 0.160 \\
\hline $\begin{array}{l}\text { Emergency } \\
\text { room }\end{array}$ & 0.40 & 0.03 & 0.34 & 0.45 & 0.44 & 0.01 & 0.41 & 0.46 & 0.04 & 0.03 & -0.02 & 0.10 & 0.212 \\
\hline $\begin{array}{l}\text { Hospital } \\
\text { outpatient }\end{array}$ & 0.89 & 0.11 & 0.67 & 1.12 & 1.15 & 0.06 & 1.04 & 1.26 & 0.25 & 0.14 & -0.02 & 0.52 & 0.065 \\
\hline $\begin{array}{l}\text { Physician's } \\
\text { visits } \\
\text { (Office based } \\
\text { visits) }\end{array}$ & 12.87 & 0.92 & 11.05 & 14.68 & 12.94 & 0.24 & 12.47 & 13.41 & 0.07 & 0.94 & -1.78 & 1.92 & 0.941 \\
\hline $\begin{array}{l}\text { Home health } \\
\text { care }\end{array}$ & 4.93 & 0.92 & 3.12 & 6.75 & 2.57 & 0.25 & 2.09 & 3.05 & -2.36 & 0.89 & -4.13 & -0.60 & 0.009 \\
\hline Prescription & 29.95 & 1.97 & 26.07 & 33.83 & 31.86 & 0.50 & 30.87 & 32.85 & 1.91 & 2.00 & -2.04 & 5.85 & 0.342 \\
\hline \multicolumn{14}{|c|}{ COPD-related visits } \\
\hline Inpatient & 0.03 & 0.01 & 0.02 & 0.04 & 0.05 & 0.01 & 0.04 & 0.06 & 0.02 & 0.01 & 0.00 & 0.03 & 0.017 \\
\hline $\begin{array}{l}\text { Emergency } \\
\text { room }\end{array}$ & 0.10 & 0.01 & 0.08 & 0.12 & 0.10 & 0.01 & 0.09 & 0.11 & 0.00 & 0.01 & -0.02 & 0.02 & 0.906 \\
\hline $\begin{array}{c}\text { Hospital } \\
\text { outpatient }\end{array}$ & 0.10 & 0.02 & 0.06 & 0.14 & 0.15 & 0.03 & 0.10 & 0.21 & 0.05 & 0.04 & -0.02 & 0.12 & 0.134 \\
\hline $\begin{array}{l}\text { Physician's } \\
\text { visits } \\
\text { (Office based } \\
\text { visits) }\end{array}$ & 2.06 & 0.41 & 1.26 & 2.86 & 1.84 & 0.07 & 1.71 & 1.97 & -0.22 & 0.42 & -1.04 & 0.61 & 0.604 \\
\hline $\begin{array}{l}\text { Home health } \\
\text { care }\end{array}$ & 0.06 & 0.02 & 0.02 & 0.11 & 0.09 & 0.01 & 0.07 & 0.11 & 0.02 & 0.02 & -0.01 & 0.06 & 0.221 \\
\hline Prescription & 1.83 & 0.08 & 1.67 & 2.00 & 2.16 & 0.04 & 2.08 & 2.24 & 0.32 & 0.09 & 0.14 & 0.50 & $<.0001$ \\
\hline
\end{tabular}

Note: ${ }^{1}$ Includes patients with zero-cost for each category.

${ }^{2}$ Adjusted by covariates including age, gender, race, educational status, region, marital status, smoking, health insurance status, poverty level, Body Mass Index, employment, Charlson Comorbidity Index.

${ }^{3}$ Significant at $\mathrm{P}<0.05$ (in bold).

CI: Confidence Interval; COPD: Chronic Obstructive Pulmonary Disease; SE: Standard Error

Table 6: Generalized Linear Model of COPD-related Medical Expenditures.

\begin{tabular}{|c|c|c|c|c|c|}
\hline Hispanic type / Demographic/Clinical Variables & Estimate $^{\mathbf{1}}$ & $\mathbf{t}$ & Lower 95\% CI $^{\text {Hispanic Type }}$ & Upper 95\% CI & p-value \\
\hline \multicolumn{7}{|c|}{} \\
\hline Non-Hispanic & Ref. & & & & \\
\hline Hispanic & -0.499 & -4.660 & -0.710 & -0.288 & $\mathbf{0 . 0 0 0}$ \\
\hline Age (continuous) & 0.005 & 1.370 & -0.002 & 0.012 & 0.173 \\
\hline
\end{tabular}




\begin{tabular}{|c|c|c|c|c|c|}
\hline \multicolumn{6}{|c|}{ Gender } \\
\hline Male & Ref. & & & & \\
\hline Female & -0.050 & -0.450 & -0.267 & 0.168 & 0.652 \\
\hline \multicolumn{6}{|c|}{ Race } \\
\hline White & Ref. & & & & \\
\hline Black & 0.102 & 0.640 & -0.214 & 0.419 & 0.525 \\
\hline Other/Multiple races & -0.166 & -1.210 & -0.438 & 0.105 & 0.229 \\
\hline \multicolumn{6}{|c|}{ Education } \\
\hline No degree or Less than high school & Ref. & $\otimes$ & $\nabla$ & $\otimes$ & $\otimes$ \\
\hline High school & -0.190 & -1.580 & -0.426 & 0.047 & 0.115 \\
\hline Some college & -0.089 & -0.670 & -0.350 & 0.173 & 0.505 \\
\hline \multicolumn{6}{|c|}{ Region } \\
\hline Northeast & Ref. & $\otimes$ & $\nabla$ & $\otimes$ & $\otimes$ \\
\hline Midwest & 0.023 & 0.130 & -0.329 & 0.376 & 0.896 \\
\hline South & -0.408 & -3.480 & -0.639 & -0.177 & 0.001 \\
\hline West & -0.270 & -2.070 & -0.526 & -0.013 & 0.039 \\
\hline \multicolumn{6}{|c|}{ Marital status } \\
\hline Married & Ref. & $\otimes$ & $\nabla$ & 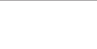 & $\nabla$ \\
\hline Unmarried & 0.264 & 2.840 & 0.081 & 0.447 & 0.005 \\
\hline Smoking & $\otimes$ & $\otimes$ & $\nabla$ & $\otimes$ & $\otimes$ \\
\hline Yes & Ref. & $\otimes$ & 凶 & $\otimes$ & $\otimes$ \\
\hline No & 0.039 & 0.360 & -0.171 & 0.248 & 0.717 \\
\hline \multicolumn{6}{|c|}{ Insurance type $\bigotimes$} \\
\hline Any private & Ref. & 凶 & 凶 & 凶 & $\otimes$ \\
\hline Public only & 0.066 & 0.800 & -0.096 & 0.229 & 0.424 \\
\hline Uninsured & -0.070 & -0.250 & -0.621 & 0.480 & 0.801 \\
\hline \multicolumn{6}{|c|}{ Poverty level $\mathbb{X}$} \\
\hline Poor/Low income & Ref. & $\nabla$ & $\nabla$ & $\otimes$ & $\otimes$ \\
\hline Middle income & -0.439 & -2.900 & -0.738 & -0.140 & 0.004 \\
\hline High income & -0.392 & -2.980 & -0.651 & -0.133 & 0.003 \\
\hline BMI (continuous) & -0.007 & -1.430 & -0.018 & 0.003 & 0.153 \\
\hline \multicolumn{6}{|c|}{ Employment》 } \\
\hline Employed & Ref. & 凶 & $\nabla$ & $\otimes$ & $\nabla$ \\
\hline Not employed & 0.365 & 3.550 & 0.163 & 0.568 & 0.000 \\
\hline CCI (continuous) & 0.396 & 8.550 & 0.305 & 0.487 & 0.000 \\
\hline
\end{tabular}

Note: ${ }^{1}$ GLM with gamma distribution and a log-link function was used.

${ }^{2}$ Significant at $\mathrm{P}<0.05$ (in bold).

CI: Confidence Interval, COPD: Chronic Obstructive Pulmonary Disease

\section{Discussion}

This study found that Hispanic patients had significantly lower overall all-cause unadjusted medical expenditures compared to Non-Hispanic patients. In addition, Hispanics also had substantially lower financial expenditures regarding COPD-related inpatient visits and prescription costs. With the exception of home health care visits, the Non-Hispanic participants exhibited more frequent use of COPD-related treatments or medications. The sample populations are intended to represent larger groups of individuals. The 2,218 Hispanic participants represent a weighted sample size of 2,363,375 people. The 9,017 Non-Hispanic participants represent a weighted sample of 22.5 million people. This study focused on the Household Component (HC) of MEPS, as mentioned in Data Source. A total of 11,235 patients were included in this 5-year MEPS data collecting period. More than half of the participants were white females with a bachelor's degree or higher. The average age of the 
participants was 55 years old, and $75 \%$ of the sample group did not smoke. The Hispanic population had a higher percentage of low income and elevated BMI than the Non-Hispanic population. However, the non-Hispanic population, on average, demonstrated higher Charlson Comorbidity Index (CCI) scores.

A Charlson Comorbidity Index (CCI) score is a measurement of concomitant disease conditions. And an elevated CCI is associated with a higher amount of health care expenditures. Hispanics who participated in the study had higher BMI values and lower average incomes, which is consistent with the previous findings. However, previous research focused on aggravating factors of COPD, rather than concomitant disease conditions. As stated by Prudente, multiple disease states can exacerbate complicated, multifactorial conditions, such as COPD $[23,24]$. Therefore, taking the Charlson Comorbidity Index into account illustrates a more realistic projection of morbidity and mortality for patients with COPD, whether Hispanic or Non-Hispanic. Based on the 9 year study of COPD by Prudente et.al, an increased number of exacerbations and CCI were linked to increased risk of mortality [24].

Prior studies have established that patients with COPD with coexisting conditions have higher health care utilization costs compared to those without coexisting medical conditions $[4,25,26]$. But there is limited information before this study about the healthcare utilization costs in Hispanic vs. non-Hispanic population with COPD. This study portrays the clear demarcation of the COPD related health care utilization costs in Hispanic and non-Hispanic people. Murphy et al. concluded that COPD has contributed to the second-highest healthcare utilization costs in the United States [25]. According to Diaz et al., healthcare utilization and access to healthcare are dependent on the insurance status of a person. The percentage of Hispanics insured in the United States is lower than the Non-Hispanics [4]. Due to the non-availability of insurances, Hispanics with COPD utilize emergency departments more frequently than clinic-based physician visits [24]. These findings are consistent with the findings of our study in regards to health care utilization, particularly with outpatient and inpatients visit costs and prescriptions. Go to states the re-admission pattern for COPD related cases was also lower in Hispanic patients compared to nonHispanic patients. This aligns with our results as we concluded that the disparity of healthcare expenditures is lower in the Hispanic population [26].

There are some limitations to this study that affect an interpretation of the results. Limitations are related to the nature of MEPS database. The database does not provide information on COPD and other respiratory disease severity, which may have an impact on the estimates. MEPS cannot provide the reasonable causal relationship between COPD and the potentially relatedcomorbidities. Only COPD-related direct medical costs were included in this study, while costs associated with comorbidities potentially related to COPD were excluded. MEPS database are self- reported rather than an objective measure, which is related to recall bias as well as under-reporting. This is the cross-sectional database and is not able to analyze an overall lifetime estimate of patientlevel expenditures. All patients with COPD and other respiratory disease from 2012 to 2016 were pooled to obtain an adequately sample size to yield reliable estimates. In the MEPS design, the samples from consecutive years may not be independent because the samples may be drawn from the same geographic region for two consecutive years. However, the MEPS documentation recommended that it is valid to keep all observations and treat them as independent because each year's data are designed to be nationally representative [23]. The procedures for complex survey sample designs in SAS and Stata were employed to ensure the accurate standard errors. This study included the only household component of MEPS consists of non-institutionalized communitydwelling residents. Patients living in supported living facilities, nursing homes, institutions, homeless people and undocumented immigrants were excluded, which limited the generality of research findings to the US population.

\section{Conclusion}

This study provided some of the first concrete financial figures concerning this healthcare disparity between ethnicities in the context of COPD-related costs among Hispanic and NonHispanic population in the US. It also revealed a possible anomaly in the healthcare system; i.e., Non-Hispanics spend substantially more on COPD-related healthcare than Hispanics. Also, the study demonstrates the necessity of assessing the Charlson Comorbidity Index (CCI) scores. These scores, which indicate compounded comorbidities, seemed to be better indications of the possible health of the participants. The results of our study demonstrate that non-Hispanics with COPD have higher healthcare utilization costs compared to Hispanics with COPD. While the overall amount spent on healthcare by Hispanics is less, it emphasizes the need for better management in this subset of the population to minimize the future harm and to establish a fair healthcare system for all Americans.

\section{References}

1. J Xu, S Murphy, D K Kochanek, B Bastian, E Arias (2018) Division of Vital Statistics. Hyattsville National Center for Health Statistics 67(5).

2. K Rabe, S Hurd, A Anzueto, P Barnes, S Buist, et al. (2007) Global strategy for the diagnosis, management, and prevention of chronic obstructive pulmonary disease. Am J Respir Crit Care Med 176(6): 532-555.

3. J Vestbo, S Hurd, A Agusti, P Jones, C Vogelmeier, et al. (2013) Global strategy for the diagnosis, management, and prevention of chronic obstructive pulmonary disease: GOLD executive summary. Am J Respir Crit Care Med 187(4): 347-365.

4. A Díaz, B Celli, JC Celedón (2018) Chronic Obstructive Pulmonary Disease in Hispanics. A 9-Year Update. Am J Respir Crit Care Med 197(1): 15-21.

5. M Drummond (2011) The hispanic paradox unraveled? Am J Respir Crit Care Med 184(11): 1222-1223.

6. S Raju, L Paulin, N Putcha, E Brigham, A Balasubramanian, et al. (2020) Rural residence is independently associated with COPD prevalence and morbidity in the national health and nutrition examination surveys (NHANES) Am J Respir Crit Care Med 201: A4863. 
7. J Davis, B Wu, D Kern, O Tunceli, K Fox, et al. (2017) Impact of Nonadherence to Inhaled Corticosteroid/LABA Therapy on COPD Exacerbation Rates and Healthcare Costs in a Commercially Insured US Population. Am Health Drug Benefits 10(2): 92-102.

8. J Brehm, J Celedón (2008) Chronic obstructive pulmonary disease in Hispanics. Am J Respir Crit Care Med 177(5): 473-478.

9. A Dhamane, C Moretz, Y Zhou, K Burslem, K saverno, et al. (2015) COPD exacerbation frequency and its association with health care resource utilization and costs. Int J Chron Obstruct Pulmon Dis 10: 2609-2618.

10. R Hummer, M Hayward (2015) Hispanic older adult health \& longevity in the united states: Current patterns \& concerns for the future. Daedalus 144(2): 20-30.

11. M Bogart, S Hopson, H Shih, R Stanford, A Coutinho (2019) Exacerbation costs associated with FF/UMEC/VI therapy in COPD compared with FF/ VI and UMEC/VI therapy. American Thoracic Society A7034.

12. M Sikirica, L Trantham, S Candrilli, D Mohan, D Neil, et al. (2018) Healthcare costs and utilization associated with muscle weakness (MW) diagnosis codes in patients with chronic obstructive pulmonary disease (COPD): A united states (US) claims analysis. American Thoracic Society A4955.

13. A Dalal, L Christensen, F Liu, A Riedel (2010) Direct costs of chronic obstructive pulmonary disease among managed care patients. Int J Chron Obstruct Pulmon Dis 5: 341-349.

14. M Borrego, M Roberts, h Petersen, A Kharat, C Blanchette (2011) Comparison of the burden and cost drivers of copd hospitalization between hispanic and white population in the united states. Value in Health 14(3): A138.

15. (2018) MEPS HC-192 2016 Full Year Consolidated Data File. Agency for Healthcare Research and Quality (AHRQ).

16. A Fischer, D Shin, D Margolis, J Takeshita (2017) Racial and ethnic differences in health care utilization for childhood eczema: An analysis of the 2001-2013 Medical Expenditure Panel Surveys. J Am Acad Dermatol 77(6): 1060-1067.

17. J Campbell, V Ghushchyan, R Mc Queen, S Cahoon Metzger, T Livington, et al. (2014) Burden of multiple sclerosis on direct, indirect costs and quality of life: National US estimates. Multiple sclerosis and related disorders 3(2): 227-236.

18. E Shirneshan, J Bailey, G Relyea, B Franklin, D Solomon, et al. (2013) Incremental direct medical expenditures associated with anxiety disorders for the U.S. adult population: evidence from the Medical Expenditure Panel Survey. J Anxiety Disord 27(7): 720-727.

19. (2019) Household Component summary tables.

20. (2019) How BLS Measures Price Change for Medical Care Services in the Consumer Price Index: US Bureau of Labor Statistics.

21. W Manning, J Mullahy J (2001) Estimating log models: to transform or not to transform? J Health Econ 20(4): 461-494.

22. (2018) Using Statistical Software Packages to Produce Estimates from MEPS Data File. Agency for Healthcare Research and Quality (AHRQ).

23. (2018) MEPS HC-036: 1996-2016 Pooled Linkage Variance Estimation File. Agency for Healthcare Research and Quality.

24. R Prudente, E Franco, C Mesquita, R Ferrari, I de Godoy, et al. (2018) Predictors of mortality in patients with COPD after 9 years. International journal of chronic obstructive pulmonary disease. 13: 3389-3398.

25. T Murphy, G Mc Avay, H Allore, J Stamm, P Simonelli (2017) Contributions of COPD, asthma, and ten comorbid conditions to health care utilization and patient-centered outcomes among US adults with obstructive airway disease. International journal of chronic obstructive pulmonary disease 12: 2515-2522.

26. T Goto, M Faridi, C Camargo Jr, K Hasegawa (2018) Time-varying readmission diagnoses during 30 days after hospitalization for COPD. Medical Care 56(8): 673-678.
ISSN: 2574-1241

\section{DOI: 10.26717/BJSTR.2020.29.004771}

Jongwha Chang. Biomed J Sci \& Tech Res

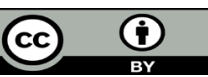

This work is licensed under Creative Commons Attribution 4.0 License

Submission Link: https://biomedres.us/submit-manuscript.php

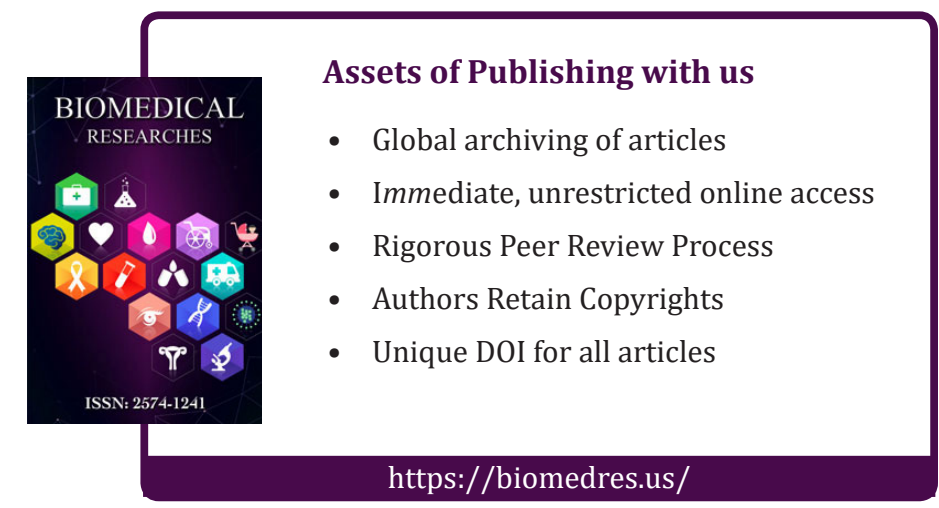

